\title{
Crucial Residues Modulating Interface of hBcl-B - hBaxBH3 Heterodimer as Probed by Computational Methods
}

\author{
Dakshinamurthy Sivakumar ${ }^{1}$; Thirunavukkarasu Sivaraman ${ }^{{ }^{*}}$. \\ ${ }^{I}$ SASTRA University, Department of Bioinformatics, Thanjavur, Tamil Nadu, India.
}

\begin{abstract}
Cancerous cells develop resistance to cell death by over expression of anti-apoptotic proteins, which are specific to interact with pro-apoptotic and BH3-only proteins of Bcl-2 family. Delineating crucial residues mediating the heterodimer complexes (anti-apoptotic proteins - pro-apoptotic/BH3-only proteins) is indispensable to develop specific antagonists to anti-apoptotic proteins. In these backgrounds, we have herein reported crucial residues of hBaxBH3 and hBcl-B (an anti-apoptotic protein specifically interacts with human Bax but does not interact with human Bak) for hetero dimerization of the polypeptides and as well validated the structural determinants of the polypeptides through variety of virtual 'alanine mutants' and 'switch mutants' by using an array of computational methods. Residues such as D53, S60, E61, K64, E69 and D71 of hBaxBH3 and R45, H50, F53, F54, Y57, M71, S74, V75, R86, V88, T89, F93 and F159 of hBcl-B were found to be crucial residues of the polypeptides for intermolecular interaction leading hetero dimerization. Moreover, 'pharmacophoric residues' for the hBaxBH3 and $h B c l-B$ have also been figured out and rationalized.
\end{abstract}

Keywords: Apoptosis, anti-apoptotic proteins, BH3-only peptides, dimer interface, dockings, virtual mutants.

"Authors for correspondence: sivaram@scbt.sastra.edu 


\section{INTRODUCTION}

Resistance to apoptosis is a common trait of cancerous cells (Hanahan and Weinberg 2000). The apoptosis is tightly regulated by proteins of Bcl-2 family, which are classified into three types: anti-apoptotic, pro-apoptotic and BH3-only proteins (Adams and Cory 1998, Reed 1998). While pro-apoptotic proteins promote apoptosis, anti-apoptotic proteins resist the apoptotic processes. The $\mathrm{BH} 3$-only proteins play essential roles on upstream and as well downstream regulations of the processes either through interactions with anti-apoptotic or pro-apoptotic proteins (Chipuk and Green 2008, Willis et al. 2007). Binding of the $\mathrm{BH} 3$-only proteins with the apoptotic proteins have been well-reported in the literature and the data suggest that the $\mathrm{BH} 3$-only proteins are highly specific to interact with antiapoptotic proteins, in particular. For instance, BH3-only proteins such as Bid, Bim and Puma have been demonstrated to interact with all antiapoptotic proteins, whereas NOXA and Hrk are shown to specifically interact with Mcl- 1 and BclXL anti-apoptotic proteins, respectively (Kim et al. 2006). Similarly, pro-apoptotic protein, Bax, is shown to interact with all six anti-apoptotic proteins characterized from homo sapiens to date, whereas Bak is interacting with all anti-apoptotic proteins other than Bcl-B (Zhai et al. 2003, Zhai et al. 2008). Since anti-apoptotic proteins have been reported to get overexpressed in all types of cancer disorders, delineating crucial residues that are structural determinants in the binding interfaces of anti-apoptotic proteins - pro-apoptotic/BH3-only proteins complexes is evidenced to be greatly helpful on designing specific antagonists to the anti-apoptotic proteins (Pinto et al. 2011, Sivakumar \& Sivaraman 2012, Sivakumar et al. 2012, Sivakumar et al. 2013).

In these backgrounds, we have probed the binding interface of a heterodimer consisting BH3-domain of human Bax (hBaxBH3, a pro-apoptotic protein) and human Bcl-B (hBcl-B, an anti-apoptotic protein) proteins in this present study. Generating theoretic structures of the complex and its validations have been elaborately discussed by the authors in an early report (Sivakumar et al. 2013). Moreover, selective binding of $\mathrm{hBclB}$ with $\mathrm{hBax}$ but not with hBak pro-apoptotic proteins have also been well documented by experimental methods (Zhai et al. 2003) and the results suggest that $\mathrm{hBclB}$ should play essential roles in regulating apoptotic processes that are exclusively governed by pro-apoptotic protein, $\mathrm{hBax}$. In this article, we have herein enumerated crucial residues of the $\mathrm{hBcl}-\mathrm{B}$ and $\mathrm{hBaxBH} 3$ that are essential for complex formation of the proteins and as well described validations of the crucial residues identified for both of the proteins through various virtual (alanine/switch) mutants of the polypeptides in conjunction with molecular docking methods in a systematic manner.

\section{MATERIALS AND METHODS}

\section{Preparation of virtual mutants of the hBcl-B and hBaxBH3}

Primary structure of $\mathrm{hBcl}-\mathrm{B}$ (Q9HD36) was retrieved from Swiss-Prot (www.uniprot.org) and was homology modeled using 2KUA (crystal structure of mouse Bcl-B). The modeling procedures and validations of the modeled threedimensional (3D) structure of the hBcl-B have been described elsewhere (Sivakumar et al. 2013). The 3D structure of 21-mer hBaxBH3 was retrieved from 2K7W PDB structure representing Bax-BIM complex (Gavathiotis et al. 2008). Various types of virtual mutants (single/triple/multiple point mutants) of the hBcl-B and $\mathrm{hBax}-\mathrm{BH} 3$ were prepared by using Swiss-Pdb Viewer (Spdbv) 4.1.0 (Guex and Peitsch 1997). In case of the hBcl-B, various residues (R45, L46, H50, F53, F54, Y57, Y60, P61, L67, M71, S74, V75, T83, G85, R86, V88, T89, F93, F159 \& F160) constituting BH3-binding groove of the protein were mutated to alanine residue only and all the virtual mutants were subjected to energy minimization followed by stereo-chemical analyses. As far as considering backbone torsion angles of the mutants with that of native $\mathrm{hBcl}-\mathrm{B}$, the structures were similar, if not identical, to each other and hence, the energy minimized virtual mutants of the hBcl-B were directly taken for molecular docking studies carried out in the present study. Whereas, in case of the hBaxBH3, various residues were mutated to alanine and as well to corresponding amino acids present in counterpart region of 20-mer human BakBH3 (hBakBH3 and structure of the polypeptide was retrieved from 2IMT). The hBaxBH3 consisting of 21 amino acids SSTMGQVGRQLAIIGDDINR ranged from 68 to 87 positions and hBakBH3 consisting of 20 amino acids QDASTKKLSECLKRIGDELDS ranged from 52 to 72 position were subjected to global sequence 
alignments using Clustal Omega (Sievers et al. 2011) and the resultant alignments were used to identify residues in corresponding positions of the two peptides. All the polypeptides (hBcl-B, $\mathrm{hBaxBH} 3$ and variants of the polypeptides) generated in the present study were subjected to energy minimization using 'steepest descent' algorithm with a tolerance of $1000 \mathrm{~kJ} / \mathrm{mol} / \mathrm{nm}$, step size of 0.01 and maximum number of minimization steps as 10,000 by means of Gromacs 4.5.1 (Hess et al. 2008).

\section{Molecular docking studies}

The wild type/virtual mutants of the hBaxBH3 were docked on BH3-binding groove of wild type/virtual mutants of the $\mathrm{hBcl}-\mathrm{B}$ by means of docking tools Hex and ClusPro 2.0 using default docking parameters (Comeau et al. 2004). However, in the molecular dockings performed using Hex, docking parameters such as search order, step size and range angles were set to be 25 , 7.5 and 180, respectively; correlation type was set as shape and electrostatics. In both docking methods, top 10 docking models were chosen for analyzing binding poses of the $\mathrm{hBaxBH} 3$ on the $\mathrm{hBcl}-\mathrm{B}$. Structural interactions of the $\mathrm{hBcl}-\mathrm{B}-$ $\mathrm{hBaxBH} 3$ complexes were scrutinized using molecular visualization tools such as VMD, PyMol 0.99 and Swiss-Pdb Viewer 4.1.0 (Delano 2002; Guex and Peitsch 1997). Herein, we would also like to point out that various molecular docking tools such as ZDOCK, PATCHDOCK, HADDOCK and GRAMM-X (in addition to the Hex and ClusPro) were also employed to study binding interaction of native $\mathrm{hBcl}-\mathrm{B}$ with native $\mathrm{hBaxBH} 3$ and as well with native hBakBH3 peptides in a benchmark study and a comprehensive analyses of the study suggested that docking results from the Hex and ClusPro were highly reliable and also in good agreement with experimental data reported for the protein peptides complexes in the literature (the $\mathrm{hBcl}-\mathrm{B}$ interacts with $\mathrm{hBaxBH} 3$ but does not interact with hBakBH3).

\section{Molecular surface properties}

Electrostatic potential maps and hydrophobic surfaces of native and variants of the $\mathrm{hBcl}-\mathrm{B}$ were prepared by using PyMol molecular visualization tool (Delano 2002). APBS module of PyMol was employed to calculate surface electrostatic potential of the protein and necessitated input files for running the module were prepared from PDB2PQR server (Dolinsky et al. 2004).
Eisenberg scale was used to calculate hydrophobic surfaces of proteins (Eisenberg et al. 1984).

\section{RESULTS AND DISCUSSION}

\section{Crucial residues of the $\mathrm{hBcl}-\mathrm{B}$ to interact with the hBaxBH3}

The Bcl-B is one of six anti-apoptotic proteins identified and structurally characterized from homo sapiens to date (Ke et al. 2001). However, the $\mathrm{hBcl}-\mathrm{B}$ is unique in its amino acid sequence and as well in biological functions: primary structure of the hBcl-B shows only about $20 \%$ sequence similarities with primary structures of other five anti-apoptotic proteins (hBcl-2, hBcl$\mathrm{XL}, \mathrm{hBcl}-\mathrm{W}$, hBfl-1 and hMcl-1); the hBcl-B specifically interacts with human Bax (hBax) but not with human Bak (hBak), whereas other five anti-apoptotic proteins interact with both $\mathrm{hBax}$ and hBak, pro-apoptotic proteins (Zhai et al. 2008). Moreover, notwithstanding similar threedimensional structures, all the 6 anti-apoptotic proteins exhibit differential binding affinities towards BH3-only proteins and as well small molecular antagonists to the proteins. Intriguingly, overexpression patterns of all the six antiapoptotic proteins are also different from each other with respect to cancerous cells (Reed 1996, Sivakumar et al. 2013). In these contexts, delineating residues that are playing essential roles in the interface of the hBcl-B - hBax complex would be emphatically useful to design highly efficient and specific small chemical molecules modulating the interface of the complex in particular.

Overall 3D structures of all the six anti-apoptotic proteins are similar to each other as documented in the literature and they have well-defined $\mathrm{BH}$ grooves and BH3-binding grooves. However, various $\mathrm{BH} 3$-only proteins and $\mathrm{BH} 3$-domain of pro-apoptotic proteins have been shown to have highly specific interactions leading to the formation of heterodimer complexes with the antiapoptotic proteins (Gavathiotis et al. 2008). In case of the $\mathrm{hBcl}-\mathrm{B}$, the $\mathrm{BH} 3$-binding groove is constituted by residues such as R45, L46, H50, F53, F54, Y57, Y60, P61, L67, M71, S74, V75, T83, G85, R86, V88, T89, F93, F159 and F160 located in helices $2,3,4 \& 5$. In other words, the BH3-binding groove is merely constituted by $\mathrm{BH} 1, \mathrm{BH} 2$ and $\mathrm{BH} 3$ domains of the protein. By replacing each of the $20 \mathrm{BH} 3$-binding groove residues with alanine one at a step, 20 single-point 
virtual mutants of the $\mathrm{hBcl}-\mathrm{B}$ were generated and energy minimized (refer methods). Binding affinities of the hBaxBH3 with each one of the 20 virtual mutants were analyzed by using Hex docking tool and Figure 1 depicts reduction in binding energies of the $\mathrm{hBaxBH} 3$ on $\mathrm{BH} 3$-binding groove of the $20 \mathrm{hBcl}-\mathrm{B}$ mutants with respect to the binding energies of wild type hBcl-B hBaxBH3 complex. By setting an arbitrary cutoff of $12 \%$ reduction in total binding energies, residues such as R45, F53, F54, V75, R86, V88, F93 and F159 were identified as crucial residues of the hBcl-B to interact with the hBaxBH3. Strikingly, the hBaxBH3 showed no binding interaction on the $\mathrm{BH} 3$-binding groove of $\mathrm{hBcl}-\mathrm{B}$ in which the eight crucial residues mentioned above were virtually mutated to alanine residue ('Crucial Ala-mutant' of the hBcl-B, Table 1). This finding suggested that the crucial residues of hBcl-B identified through 'virtual alanine scanning' strategy are quite reliable as evinced herein. It should be mentioned that 3D conformations of native $\mathrm{hBcl}-\mathrm{B}$ and its virtual mutants were essentially same except a subtle structural changes at the respective site of residue chosen for alanine substitution mutation in each case. In other words, it is presumed that alanine mutants of the $\mathrm{hBcl}-\mathrm{B}$ are capable of adopting compact 3D structures as if native hBcl-B adopts its 3D folds. However, effect of the residues chosen for alanine substitution mutation on folding pathways of the proteins can be unambiguously understood by using protein folding experiments only. In general, unless until the protein mutants adopt native 3D folds conformations, the generated protein mutants will not be useful for the binding studies even by experimental methods. Notwithstanding this inherent limitation of the in silico studies on understanding the relationships between protein mutants and folding pathways, virtual protein mutants have been successfully used in drug designing strategies and as well to understand structural interactions of various complexes of protein mutants as reported in the literature (Goh et al. 2002, Anthony et al. 2015, Nagasundaram et al. 2015).

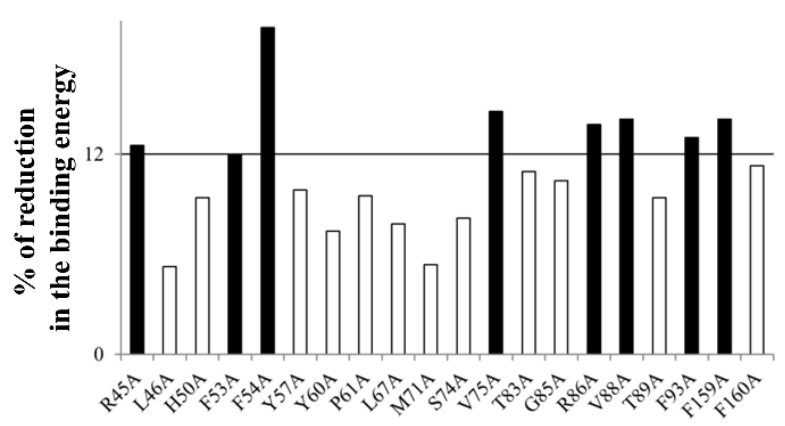

Figure 1.Percentage of reductions in the docking energies of the hBaxBH3 peptide to interact on the $\mathrm{BH} 3-$ binding groove of various single point alanine substitution mutants of the hBcl-B is shown. Percentage of reduction in the energies of hBcl-B mutants hBaxBH3 complexes were calculated with respect to docking energy $(-611 \mathrm{kcal} / \mathrm{mol})$ of native $\mathrm{hBcl}-\mathrm{B}$ $\mathrm{hBaxBH} 3$ complex. Crucial residues of the hBcl-B to interact with the $\mathrm{hBaxBH} 3$ were identified by setting an arbitrary cutoff at $12 \%$ of the reduction binding energies.

The crucial residues of the $\mathrm{hBcl}-\mathrm{B}$ figured out in the previous paragraph were from molecular docking experiments performed under 'static' conditions as described. It is also worthy of mentioning that crucial residues of the $\mathrm{hBcl}-\mathrm{B}$ to interact with $\mathrm{hBaxBH} 3$ have also been recently mapped out under 'dynamic' conditions by means of 'peptidodynmimetic' computational method and the data revealed that residues such as $\mathrm{H} 50, \mathrm{~F} 53$, Y57, M71, S74, V75, T89 and F93 were interfacedeterminants of the protein (Sivakumar et al. 2013). In the 'peptidodynmimetic' method, temperature-dependent molecular dynamics simulations of the hBcl-B - hBaxBH3 complex structure were carried out in near-physiological

Table 1- Interactions between $\mathrm{hBaxBH} 3$ and various virtual mutants of hBcl-B. Binding interaction between the two polypeptides were studied by using Hex/ClusPro molecular docking tools

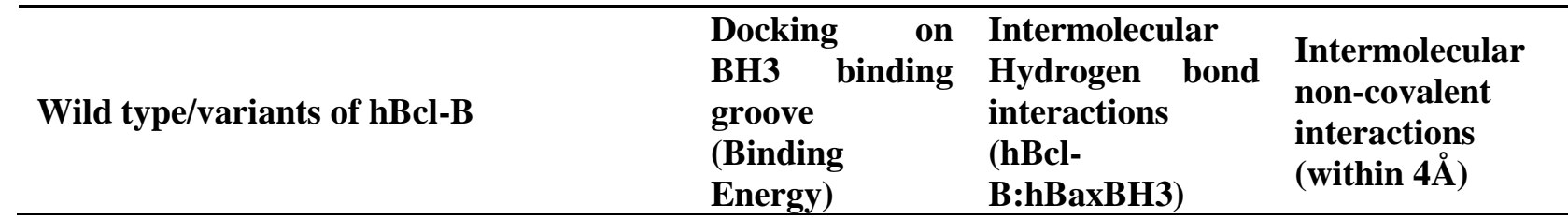




\begin{tabular}{|c|c|c|c|c|}
\hline \multicolumn{5}{|l|}{ Wild type hBcl-B } \\
\hline & \multicolumn{2}{|c|}{$\begin{array}{l}\text { Docked } \\
(-611 \mathrm{kcal} / \mathrm{mol})\end{array}$} & $\begin{array}{l}\text { H50:HD1-O:E63 } \\
\text { Y57:HH-O:T56 } \\
\text { Y57:HH- } \\
\text { OG1:T56 } \\
\text { S74:HG-OE1:E61 } \\
\text { S74:HG-OE2:E61 } \\
\text { T89:H-OD2:D68 } \\
\text { Y57:OH- } \\
\text { HG1:T56 } \\
\text { Y57:OH-H:K57 } \\
\text { M71:SD- } \\
\text { HZ1:K64 } \\
\text { M71:SD- } \\
\text { HZ2:K64 } \\
\text { M71:SD- } \\
\text { HZ3:K64 } \\
\text { S74:OG- } \\
\text { HH12:R65 } \\
\text { S74:O-HH22:R65 }\end{array}$ & $\begin{array}{lll}\text { A42, } & \text { R45, } & \text { L46, } \\
\text { H50, } & \text { F53, } & \text { F54, } \\
\text { Y57, Y60, } & \text { P61, } \\
\text { L67, M71, S74, } \\
\text { V75, T83, G85, } \\
\text { R86, V88, T89, } \\
\text { F93, F159, F160 }\end{array}$ \\
\hline $\begin{array}{l}\text { 'Crucial Ala-mutant' of hBcl-B } \\
\text { (R45A, F53A, F54A, V75A, R86A, V88A, } \\
\text { F93A, F159A) }\end{array}$ & \multicolumn{2}{|c|}{ Undocked } & NA & NA \\
\hline $\begin{array}{l}\text { 'Peptidodynmimetic Ala-mutant' of hBcl-B } \\
\text { (H50A, F53A, Y57A, M71A, S74A, V75A, } \\
\text { T89A, F93A) }\end{array}$ & \multicolumn{2}{|c|}{$\begin{array}{l}\text { Non-specifically } \\
\text { Docked }\end{array}$} & $\begin{array}{l}\text { Y60:O-HZ1:K57 } \\
\text { Y60:O-HZ3:K57 }\end{array}$ & $\begin{array}{lll}\text { L46, } & \text { A53, } & \text { F54, } \\
\text { A56, } & \text { A57, } & \text { Y60, } \\
\text { P61, } & \text { L67, } & \text { L70, } \\
\text { A71, } & \text { A74, } & \text { A75, } \\
\text { T83, } & \text { G85, } & \text { R86, } \\
\text { A89, } & \text { L90, } & \text { A93, } \\
\text { L97 } & \end{array}$ \\
\hline $\begin{array}{l}\text { 'Tredecuple Ala-mutant' of hBcl-B } \\
\text { (R45A, H50A, F53A, F54A, Y57A, M71A, } \\
\text { S74A, V75A, R86A, V88A, T89, F93A, } \\
\text { F159A) }\end{array}$ & \multicolumn{2}{|c|}{ Undocked } & NA & NA \\
\hline \multicolumn{5}{|c|}{ 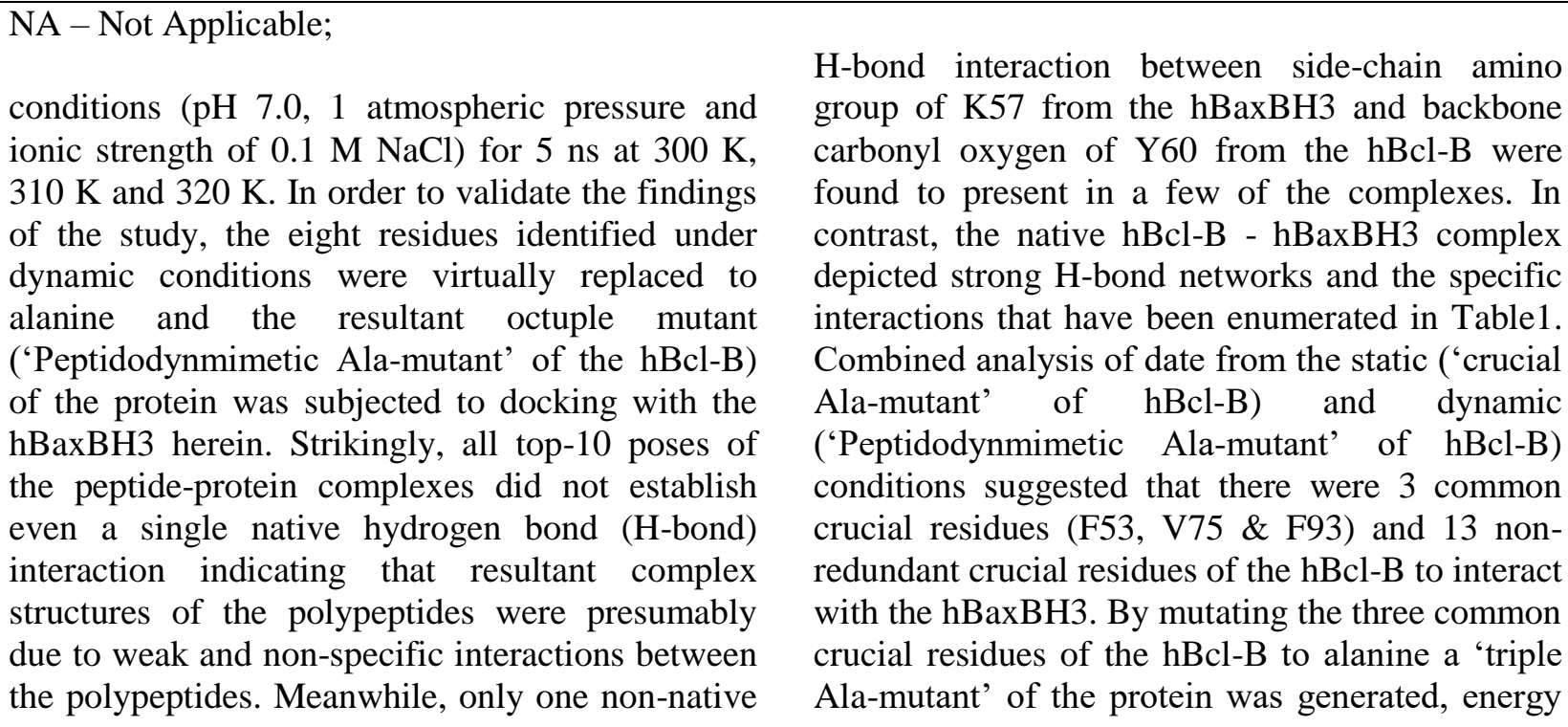 } \\
\hline
\end{tabular}


minimized to the level of structural convergence and subjected to docking with the hBaxBH3. However, the docking outputs were not straightforward as the 'triple Ala-mutant' hBcl-B $\mathrm{hBaxBH} 3$ complex formations were of mixed docking poses: out of 10 top poses selected, only 4 poses depicted that the 'triple Ala-mutant' hBcl-B was capable of accommodating the $\mathrm{hBaxBH} 3$ on the $\mathrm{BH} 3$-binding groove with most of native intermolecular interactions, whereas other 6 poses suggested that interactions of the $\mathrm{hBaxBH} 3$ with $\mathrm{hBcl}-\mathrm{B}$ were not on the $\mathrm{BH} 3$-binding groove of the protein suggesting non-specific complex formations. These results imply that absence of the three common crucial residues along with other residues as detected either under 'static' or 'dynamic' conditions is obvious prerequisite in order to entirely preclude complex formations between the hBcl-B and hBaxBH3. Evidently, the $\mathrm{hBcl}-\mathrm{B}$ obtained upon replacing side-chains of the 13 crucial residues to methyl groups did not interact with the hBaxBH3 ('Tredecuple Alamutant' of hBcl-B, Table 1). These differential interactions of the $\mathrm{hBaxBH} 3$ with the native $\mathrm{hBcl}-$ $\mathrm{B}$ and the 'tredecuple Ala-mutant' of $\mathrm{hBcl}-\mathrm{B}$ could be attributed to differences in the BH3-binding grooves of the proteins (Fig. 2). The grooves of the two proteins were found to be different from each other in terms of geometrical orientations, electrostatic surfaces and distributions of hydrophobic batches. In these backgrounds, the data described above suggested that residues such as R45, H50, F53, F54, Y57, M71, S74, V75, R86, V88, T89, F93 and F159 of the hBcl-B play essential contributions to tether the $\mathrm{hBaxBH} 3$ peptide on the BH3-binding groove of the $\mathrm{hBcl}-\mathrm{B}$ through a specific network of non-covalent interactions.

\section{Crucial residues of the $\mathrm{hBaxBH} 3$ to interact with the hBcl-B}

All six anti-apoptotic proteins bind with Bax protein from Homo sapiens as reported in the literature and 3D structures of Bcl-2 (2XAO), Bcl$\mathrm{XL}$ (3PL7) and Mcl-1 (3PK1) bound with BaxBH3 peptides have also been deposited in PDB database (Ku et al. 2011, Czabotar et al. 2011). Zhai et al. (2003) have analyzed the binding affinities for a series alanine substituted $\mathrm{hBaxBH} 3$ peptides with $\mathrm{hBcl}-\mathrm{B}$ and demonstrated that Leu70 of the hBaxBH3 was most crucial amino acid governing binding to the protein. We have recently identified residues of the $\mathrm{hBaxBH} 3$ that are essential for interacting with $\mathrm{hBcl}-\mathrm{B}$ by using temperature-dependent molecular dynamics simulations ('peptidodynmimetic' method mentioned above) in a stringent manner and crucial residues of the peptide were found to be D53, S60, E61, K64, E69 and D71 (Sivakumar et al. 2013). Of the six residues, S60, E61 \& K64 are denoted as 'pharmacophoric residues' (essential to design small chemical molecules mimicking the $\mathrm{hBaxBH} 3$ interactions with the $\mathrm{hBcl}-\mathrm{B}$ ) and D53, E69 \& D71 are denoted as ' $\mathrm{CN}$ tethering residues' (essential to tether $\mathrm{C}$ - and $\mathrm{N}$-termini of the $\mathrm{hBaxBH} 3$ on $\mathrm{BH} 3$-binding region of the $\mathrm{hBcl}-\mathrm{B}$ ). However, validations of the crucial residues identified by the MD simulations have not yet been systematically addressed. Herein, we generated sextuple ('Crucial Ala-mutant') and two types of triple points mutants ('Pharmacophoric Ala-mutant' and ' $\mathrm{CN}$ tethering Ala-mutant') of the $\mathrm{hBaxBH} 3$ by alanine substitution strategy and then the mutants were subjected to molecular dockings with the hBcl-B protein (Table 2). The docking data suggested that all the three mutants were ineffective to bind on the $\mathrm{BH} 3$-binding groove of the $\mathrm{hBcl}-\mathrm{B}$ indicating the six residues of the $\mathrm{hBaxBH} 3$ screened by the MD simulations are absolutely prerequisite to form complex with the $\mathrm{hBcl}-\mathrm{B}$. In other words, either in the absence of 'pharmacophoric' residues (S60, E61 \& K64) or in the absence of ' $\mathrm{CN}$ tethering' residues (D53, E69 \& D71), the hBaxBH3 was found to be unable to interact with the $\mathrm{hBcl}-\mathrm{B}$.

It has been unambiguously authenticated that the $\mathrm{hBcl}-\mathrm{B}$ does not bind with the $\mathrm{hBakBH} 3$ by using experimental and as well by computational methods (Ke et al. 2001, Zhai et al. 2003, Zhai et al. 2008, Sivakumar et al. 2013). The 20-mer hBakBH3 (SSTMGQVGRQLAIIGDDINR) and 21-mer $\mathrm{hBaxBH} 3$ (QDASTKKLSECLKRIGDELDS) showed 52\% sequence similarity and similar 3D structures (Fig. 3and refer methods). Moreover, the two peptides depicted global sequence alignment with just a gap at first amino acid of the $\mathrm{hBakBH} 3$ (as sequence length of $\mathrm{hBaxBH} 3$ is one amino acid longer than that of hBakBH3). On the basis of the sequence alignments, we prepared three switch mutants of the hBaxBH3: 'Crucial Switch-mutant', 'Pharmacophoric Switch-mutant' and 'CN tethering Switch-mutant' (Table 2). In all the cases, target residues of the $\mathrm{hBaxBH} 3$ were substituted to the residues present in the corresponding positions of the $\mathrm{hBakBH} 3$. If six crucial residues of the $\mathrm{hBaxBH} 3$ identified by MD 
simulations were sole responsible on governing binding between the $\mathrm{hBcl}-\mathrm{B}$ and $\mathrm{hBaxBH} 3$, the 'Crucial Switch-mutant' must completely nullify interaction with the hBcl-B. Strikingly, the 'Crucial Switch-mutant' of hBaxBH3 did not dock on the BH3-binding groove of $\mathrm{hBcl}-\mathrm{B}$ (Table2). More importantly, the other two substitution mutants ('Pharmacophoric' and ' $\mathrm{CN}$ tethering') of the $\mathrm{hBaxBH} 3$ were also showed no binding with the hBcl-B strongly supporting that the residues mapped out by MD simulations are prerequisite residues of the $\mathrm{hBaxBH} 3$ to interact with the $\mathrm{hBcl}-$ B. Moreover, the docking results of 'Switchmutants' of the hBaxBH3 are also in excellent agreement with docking results of 'Ala-mutants' of the $\mathrm{hBaxBH} 3$ described in the previous paragraph (Table 2). To further confirm the specific interaction between the $\mathrm{hBaxBH} 3$ and $\mathrm{hBcl}-\mathrm{B}$, all residues but other than 6 crucial residues of the $\mathrm{hBaxBH} 3$ were replaced with residues that are present in the corresponding position of the $\mathrm{hBakBH} 3$ and the resultant mutant was denoted as 'Non-crucial Switch-mutant' of the hBaxBH3 (Table 2). The 'Non- crucial Switchmutant' was found to bind on the BH3-binding groove of $\mathrm{hBcl}-\mathrm{B}$ and binding energy was found to be $-438 \mathrm{kcal} / \mathrm{mol}$. Residues such as R45, L46, V75, T83, W84, G85, R86, V88, T89, F159, F160, and R161 of the hBcl-B were found to present within $4 \AA$ proximity of the $\mathrm{hBaxBH} 3$ (Table 2). Residues such

Table 2. Interactions between $\mathrm{hBcl}-\mathrm{B}$ and various virtual mutants of $\mathrm{hBaxBH}$. Binding interaction between the two polypeptides were studied by using Hex/Cluspro molecular docking tools

\begin{tabular}{|c|c|c|c|}
\hline Wild type/variants of hBaxBH3 & $\begin{array}{l}\text { Docking on } \\
\text { BH3 binding } \\
\text { groove } \\
\text { (Binding } \\
\text { Energy) } \\
\end{array}$ & $\begin{array}{l}\text { Intermolecular } \\
\text { Hydrogen bond } \\
\text { interactions } \\
(\text { hBaxBH3:hBcl-B) }\end{array}$ & $\begin{array}{l}\text { Intermolecular } \\
\text { non-covalent } \\
\text { interactions } \\
\text { (within } 4 \AA \text { ) }\end{array}$ \\
\hline $\begin{array}{l}\text { 'Crucial Ala-mutant' of hBaxBH3 } \\
\text { (D53A, S60A, E61A, K64A, E69A, } \\
\text { D71A) }\end{array}$ & Undocked & NA & NA \\
\hline $\begin{array}{l}\text { 'Pharmacophoric Ala-mutant' of } \\
\text { hBaxBH3 } \\
\text { (S60A. E61A, K64A) }\end{array}$ & Undocked & NA & NA \\
\hline $\begin{array}{l}\text { 'CN tethering Ala-mutant' of hBaxBH3 } \\
\text { (D53A, E69A, D71A) }\end{array}$ & Undocked & NA & NA \\
\hline $\begin{array}{l}\text { 'Crucial Switch-mutant' of hBaxBH3 } \\
\text { (D53S, S60G, E61R, K64A, E69D, } \\
\text { D71N) }\end{array}$ & Undocked & NA & NA \\
\hline $\begin{array}{l}\text { 'Pharmacophoric Switch-mutant' of } \\
\text { hBaxBH3 (S60G, E61R, K64A) }\end{array}$ & Undocked & NA & NA \\
\hline $\begin{array}{l}\text { 'CN tethering Switch-mutant' of } \\
\text { hBaxBH3 } \\
\text { (D53S, E69D, D71N) }\end{array}$ & Undocked & NA & NA \\
\hline $\begin{array}{l}\text { 'Non-Crucial Switch-mutant' of } \\
\text { hBaxBH3 } \\
\text { (A54S, S55T, T56M, K57G, K58Q, } \\
\text { L59V, C62Q, R65I, L70I, S72R) }\end{array}$ & $\begin{array}{l}\text { Docked } \\
(-438 \mathrm{kcal} / \mathrm{mol})\end{array}$ & $\begin{array}{l}\text { D68:OD-HG1:T83 } \\
\text { D68:OD-HG1:T83 } \\
\text { D71:O-HH21:R86 } \\
\text { D71:O-HH22:R86 } \\
\text { R72:HH11-O:G85 }\end{array}$ & $\begin{array}{l}\text { R45, L46, V75, } \\
\text { T83, W84, G85, } \\
\text { R86, V88, T89, } \\
\text { F159, F160, R161 }\end{array}$ \\
\hline \multicolumn{3}{|c|}{$\begin{array}{l}\text { NA - Not Applicable } \\
\text { as D68, D71 and R72 present in C-terminal of the } \\
\text { 'Non-crucial Switch-mutant' hBaxBH3 showed H- } \\
\text { bond (hydrogen bonding) interactions with T83, } \\
\text { R86 and G85 (present in the BH3-binding groove) } \\
\text { of the hBcl-B (Table 2, Supplementary Figure S1), }\end{array}$} & $\begin{array}{l}\text { ninuses of both } \\
\text { a-crucial Switch- } \\
\text { ound to have a } \\
\text { the BH3-binding } \\
\text { able 1) and these } \\
\text { to presence of }\end{array}$ \\
\hline
\end{tabular}


structurally and as well chemically similar residues in C-terminuses of the two peptides, whereas $\mathrm{N}$-terminuses of the two polypeptides were drastically differed from each other (Fig. $3 \&$ Fig. 5). These striking data have clearly revealed that D53, S60, E61, K64, E69 and D71 of the $\mathrm{hBaxBH} 3$ are essential for its binding to the $\mathrm{hBcl}-$ $\mathrm{B}$ anti-apoptotic protein. Moreover, the data have also brought a rationalization for the differential interactions of the $\mathrm{hBaxBH} 3$ and $\mathrm{hBakBH} 3$ towards the hBcl-B: while the native hBaxBH3 and 'Non-crucial Switch-mutant' of the hBaxBH3 showed binding with the $\mathrm{hBcl}-\mathrm{B}$, the native $\mathrm{hBakBH} 3$ lacks binding affinity with the hBcl-B as the $\mathrm{hBakBH} 3$ does not have the six crucial residues at appropriate positions (Fig. 5). It is also worthy of mentioning that free energy of binding for various virtual mutants of $\mathrm{hBaxBH} 3$ bound with the native $\mathrm{hBcl}-\mathrm{B}$ and similarly, various virtual mutants of $\mathrm{hBcl}-\mathrm{B}$ bound with native hBaxBH3 can be estimated using computational methods such as 'umbrella sampling', 'thermodynamic integration', 'alchemical freeenergy perturbation', MM-PBSA, MM-GBSA and QM-MM techniques (Michel \& Essex 2010, Steinbrecher \& Labahn 2010, Genheden \& Ryde 2015 ) in near future and such studies (though the methods are computationally expensive and challenging for the protein - peptide complexes) would bring some valuable quantitative thermodynamic parameters to the results presented herein from molecular docking studies on the $\mathrm{hBcl}-\mathrm{B}-\mathrm{hBaxBH} 3$ complexes.
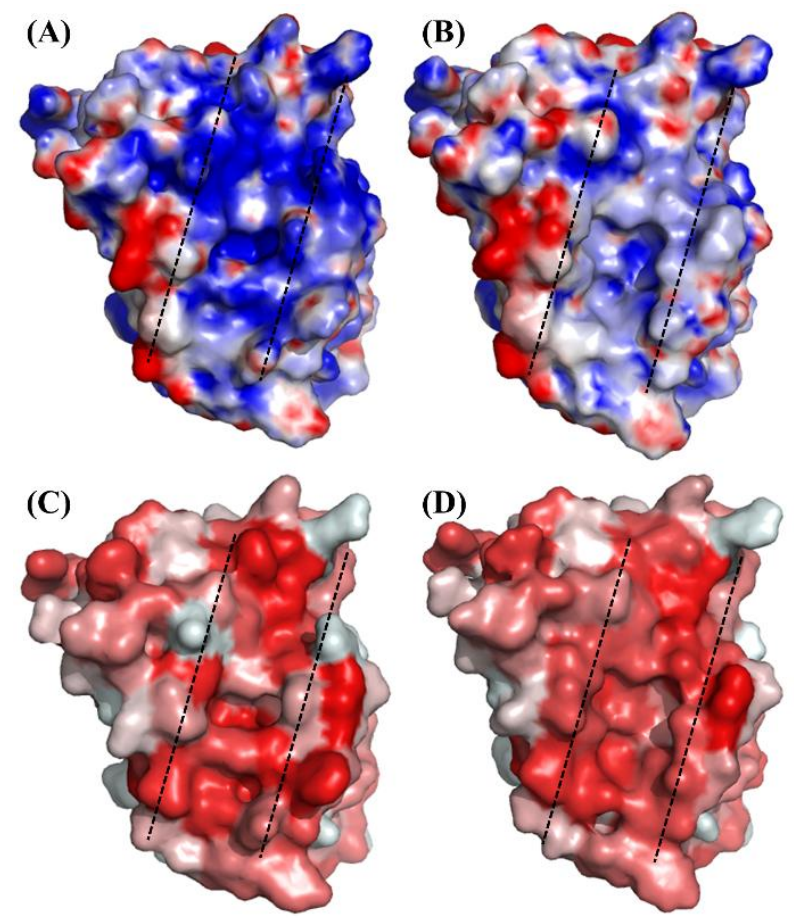

Figure 2.Electrostatic potential surface and hydrophobic surface of wild-type hBcl-B are represented in a) \& c) respectively and the same for 'Tredecuple Ala-mutant' of the hBcl-B (refer text) are represented in b) \& d), respectively. BH3-binding grooves of the proteins are outlined by a pair of dashed lines (readers are referred to the web version of this article for color illustrations of the figure).

\section{A)}
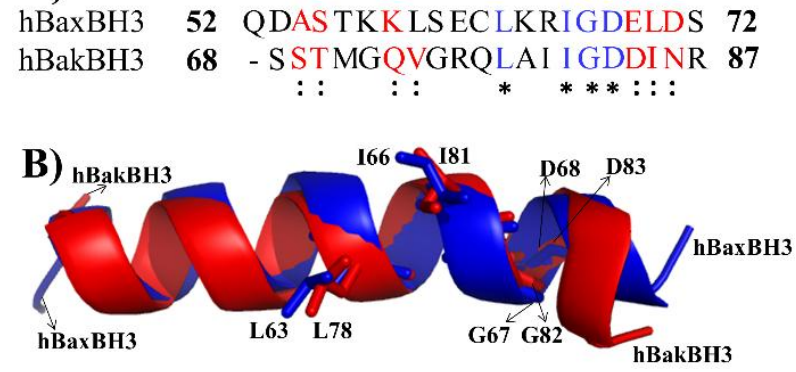

Figure 3.Sequence and structural alignments of the $\mathrm{hBaxBH} 3$ and $\mathrm{hBakBH} 3$ peptides. a) Identical and similar residues are marked by asterisk $(*)$ and colon $(:)$, respectively, in the sequence alignment of the $\mathrm{hBaxBH} 3$ and hBakBH3. b) Side chain orientations of four identical residues present in the two peptides as per the structural alignment are shown in sticks (readers are referred to the web version of this article for color illustrations of the figure). 


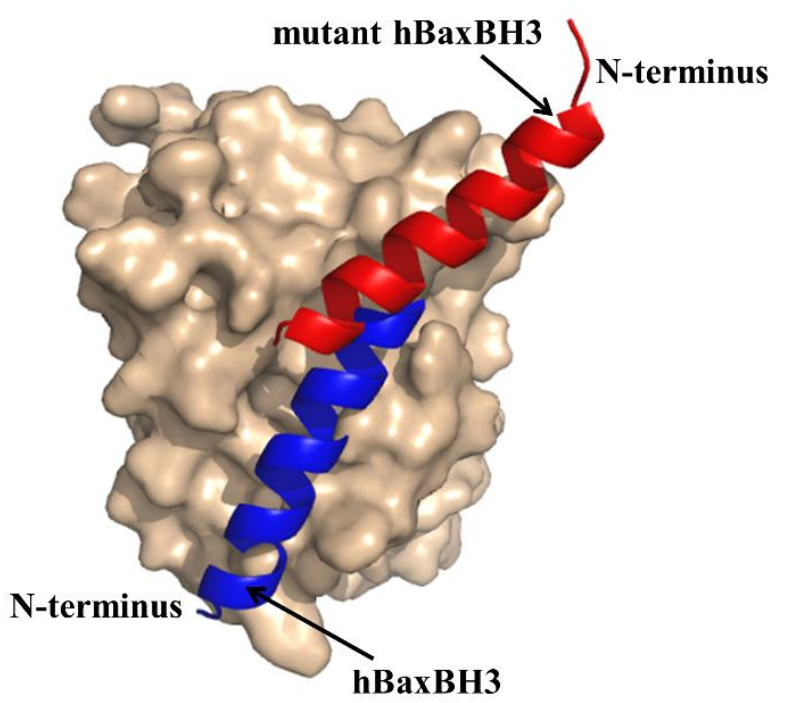

Figure 4.Docked conformations of wild type hBaxBH3 and 'Non-Crucial Switch-mutant' of the hBaxBH3 on $\mathrm{BH} 3-$ binding groove of the $\mathrm{hBcl}-\mathrm{B}$ are depicted. The $\mathrm{hBaxBH} 3$ peptides and $\mathrm{hBcl}-\mathrm{B}$ are represented in cartoon and surface models, respectively (readers are referred to the web version of this article for color illustrations of the figure).
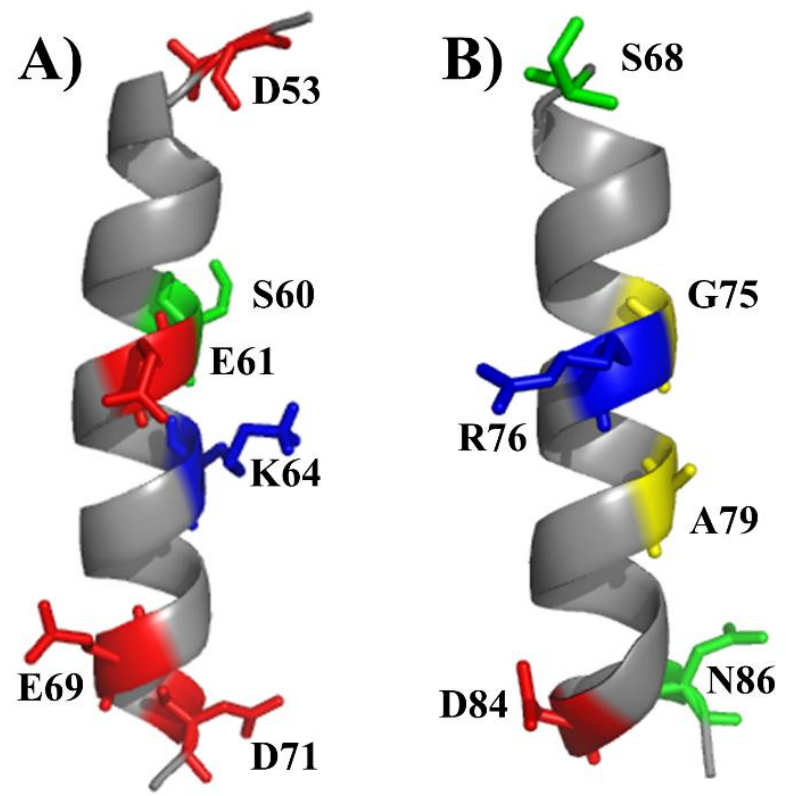

Figure 5.'Pharmacophoric' (S60, E61 \& K64) residues and 'CN tethering' (D53, E69 \& D71) residues of a) the $\mathrm{hBaxBH} 3$ and residues present in the corresponding positions of $b$ ) the $\mathrm{hBakBH} 3$ are illustrated in sticks (readers are referred to the web version of this article for color illustrations of the figure).

\section{CONCLUSIONS}

In summary, crucial residues and as well pharmacophoric residues of the $\mathrm{hBcl}-\mathrm{B}$ and hBaxBH3 to interact each other have been identified and also validated by using various virtual mutants of the polypeptides in conjunction with molecular docking strategies. While residues such as R45, H50, F53, F54, Y57, M71, S74, V75, R86, V88, T89, F93 and F159 could be identified as crucial residues of the $\mathrm{hBcl}-\mathrm{B}$ for complex formation with the $\mathrm{hBaxBH} 3$, residues such as F53, V75 and F93 could be defined as 'pharmacophoric residues' of the protein as the three residues were characterized to be indispensable for the hBcl-B - hBaxBH3 complex formation under both 'static' and 'dynamic' conditions. In case of hBaxBH3, residues such as D53, S60, E61, K64, E69 and D71 were found to be crucial for hetero dimerization of the $\mathrm{hBcl}-\mathrm{B}-$ $\mathrm{hBaxBH3}$. The six residues could be further divided into two categories: ' $\mathrm{CN}$ tethering' residues (D53, E69 and D71) and 'pharmacophoric residues' (S60, E61 and K64). In addition, the data presented in the present studies have also enlightened a rationalization for differential interactions of the $\mathrm{hBaxBH} 3$ and $\mathrm{hBakBH} 3$ on $\mathrm{BH} 3$-binding groove of the $\mathrm{hBcl}-\mathrm{B}$ from structural standpoints for the first time: residues such as S68, G75, R76, A79, D84 \& N86 of the hBakBH3 could preclude its interaction with the $\mathrm{hBcl}-\mathrm{B}$, whereas residues such as D53, S60, E61, K64, E69 \& D71 of the hBaxBH3 could be primarily responsible to tether the peptide on $\mathrm{BH} 3$-binding groove of the anti-apoptotic protein, hBcl-B.

\section{REFERENCES}

Adams JM, Cory S. The Bcl-2 protein family: arbiters of cell survival. Science. 1998; 281: 1322-1326.

Anthony J, Rangamaran VR, Gopal D, Sivashankarasubbiah KT, Leema M, Thilagam J, et al. Ultraviolet and 5'Fluorodeoxyuridine induced random mutagenesis in Chlorella vulgaris and its impact on fatty acid profile: A new insight on lipid metabolizing genes and its structural characterization of related proteins. Mar Biotechnol. 2015; $17: 66-80$.

Chipuk JE, Green DR. How do BCL-2 proteins induce mitochondrial outer membrane permeabilization?.Trends Cell Biol. 2008; 18: 157164.

Comeau SR, Gatchell DW, Vajda S, Camacho CJ. ClusPro: a fully automated algorithm for proteinprotein docking. Nuc Acids Res. 2004; 32: W96-99.

Czabotar PE, Lee EF, Thompson GV, Wardak AZ, Fairlie WD, Colman PM. Mutation to Bax beyond the BH3 domain disrupts interactions with pro- 
survival proteins and promotes apoptosis. $J$ Biol Chem. 2011; 286: 7123-7131.

Delano WL. The PyMOL Molecular Graphics System (2002) DeLano Scientific, San Carlos, CA, USA. http://www.pymol.org.

Dolinsky TJ, Nielsen JE, McCammon JA, Baker NA. PDB2PQR: an automated pipeline for the setup, execution, and analysis of Poisson-Boltzmann electrostatics calculations. Nucleic Acids Res. 2004; 32: W665-W667.

Eisenberg D, Schwarz E, Komaromy M, Wall R. Analysis of membrane and surface protein sequences with the hydrophobic moment plot. $\mathrm{J} \mathrm{Mol}$ Biol. 1984; 179:125-142.

Gavathiotis E, Suzuki M, Davis ML, Pitter K, Bird GH, Katz SG, et al. BAX activation is initiated at a novel interaction site. Nature. 2008; 455:1076-1081.

Genheden S, Ryde U. The MM/PBSA and MM/GBSA methods to estimate ligand-binding affinities. Expert Opin Drug Discov. 2015;10: 449-461.

Goh YY, Frecer V, Ho B, Ding JL. Rational design of green fluorescent protein mutants as biosensors for bacterial endotoxin. Protein Eng. 2002; 15: 493502.

Guex N, Peitsch MC. SWISS-MODEL and the SwissPdbViewer: an environment for comparative protein modeling. Electrophoresis. 1997; 18: 2714-2723.

Hanahan D, Weinberg RA. Hallmarks of Cancer: The Next Generation. Cell. 2000; 100: 57-70.

Hess B, Kutzner C, Spoel DVD, Lindahl E. GROMACS 4: Algorithms for Highly Efficient, Load-Balanced, and Scalable Molecular Simulation. J Chem Theory Comput. 2008; 4: 435-447.

Kim H, Rafiuddin-Shah M, Tu H-C, Jeffers JR, Zambetti GP, Hsieh JJ-D, Cheng EH-Y. Hierarchical regulation of mitochondrion-dependent apoptosis by BCL-2 subfamilies. Nat Cell Biol. 2006; 8: 1348-1358.

Ke N, Godzik A, Reed JC. Bcl-B, a Novel Bcl-2 family member that differentially binds and regulates Bax and Bak. J Biol Chem. 2001; 276: 12481-12484.

$\mathrm{Ku}$ B, Liang C, Jung JU, Oh BH. Evidence that inhibition of BAX activation by BCL-2 involves its tight and preferential interaction with the $\mathrm{BH} 3$ domain of BAX. Cell Res. 2011; 21: 627-641.

Michel J, Essex JW. Prediction of protein-ligand binding affinity by free energy simulations: assumptions, pitfalls and expectations. J Comput Aided Mol Des. 2010; 24: 639-658.

Nagasundaram N, Zhu H, Liu J, Karthick V, George PDC, Chiranjib C, Luonan C. Analyzing the effect of mutation on protein function and discovering potential inhibitors of CDK4: molecular mdelling and dynamics studies. PLoS ONE. 2015; 10: e0133969.

Pinto M, Orzaez MDM, Delgado-Soler L, Perez JJ, Rubio-Martinez J. Rational Design of New Class of
BH3-Mimetics As Inhibitors of the Bcl-xL Protein. J Chem Inf Model. 2011; 51: 1249-1258.

Reed JC. Mechanisms of Bcl-2 family protein function and dysfunction in health and disease. Behring Inst Mitt. 1996; 97: 72-100.

Reed JC. Bcl-2 family proteins. Oncogene. 1998; 17: 3225-3236.

Sievers F, Wilm A, Dineen D, Gibson TJ, Karplus K, Li $\mathrm{W}$, et al. Fast, scalable generation of high-quality protein multiple sequence alignments using Clustal Omega. Mol Syst Biol. 2011; 7:539-544.

Sivakumar D, Sivaraman T. Designing of de novo dual inhibitors for Bcl-XL and Mcl-1 of Bcl2-family proteins by computational methods. IEEEInternational Conference on Advances in Engineering, Science and Management, ICAESM. 2012; art. no. 6216310 , 174-78.

Sivakumar D, Richa T, Rajesh SS, Gorai B, Sivaraman T. In silico methods for designing antagonists to anti-apoptotic members of Bcl-2 family proteins. Mini Rev Med Chem. 2012; 12: 1144-1153.

Sivakumar D, Gorai B, Sivaraman T. Screening efficient BH3-mimetics to $\mathrm{hBcl}-\mathrm{B}$ by means of peptidodynmimetic method. Mol BioSyst. 2013; 9: 700-712.

Steinbrecher T, Labahn A. Towards accurate free energy calculations in ligand protein-binding studies. Current Medicinal Chemistry, 2010; 17: 767-785.

Willis SN, Fletcher JI, Kaufmann T, Delft MFV, Chen L, Czabotar PE, et al. Apoptosis Initiated When BH3 Ligands Engage Multiple Bcl-2 Homologs, Not Bax or Bak. Science. 2007; 315: 856-859.

Zhai D, Ning KE, Zhang H, Ladror U, Joseph M, Eichinger A, et al. Characterization of the antiapoptotic mechanism of Bcl-B. Biochem J. 2003; 376: 229-236.

Zhai D, Jin C, Huang Z, Satterthwait AC, Reed JC. Differential Regulation of Bax and Bak by Antiapoptotic Bcl-2 Family Proteins Bcl-B and Mcl-1. J Biol Chem. 2008; 283: 9580-9586.

Received: May07, 2016; Accepted: June01, 2016 